\title{
Improved MMSE vector precoding based on the MBER criterion
}

\author{
W. Yao, S. Chen and L. Hanzo \\ School of ECS, University of Southampton, SO17 1BJ, United Kingdom \\ E-mails: \{wy07r, sqc, lh\}@ecs.soton.ac.uk, http://www-mobile.ecs.soton.ac.uk
}

\begin{abstract}
A novel vector precoding scheme is proposed for the downlink of a multiuser system equipped with multiple antennas transmitting to single-antenna aided mobile receivers. Our transmit preprocessing scheme first invokes a regularized channel inversion and then superimposes a perturbation vector to directly minimize the Bit Error Ratio (BER) of the system as an improvement to the well-known Minimum Mean-SquareError (MMSE) vector precoding scheme. Our simulation results demonstrate that the proposed vector precoding scheme acheives the same BER performance as the MMSE vector precoding at the same complexity, when only discrete vector perturbations are allowed. However, the performance of the proposed vector precoding scheme can be further improved based on the MBER criterion, when continuous-valued vector perturbations are carried out.
\end{abstract}

\section{INTRODUCTION}

In the downlink (DL) of a Space-Division Multiple-Access (SDMA) system non-cooperative Mobile Stations (MS) are unable to employ Multiuser Detection (MUD) for mitigating the multiuser interference. In order to facilitate the employment of a low-complexity single-user receiver, the transmitted multiuser DL signals may be pre-processed at the Base Station (BS), exploiting the knowledge of each user's unique Channel Impulse Response (CIR) for differentiating their transmissions, leading to the appealing concept of Multiuser Transmission (MUT) [1]. Naturally, this is only possible, if accurate Channel State Information (CSI) is available at the transmitter. The assumption that the DL CIR is known at the BS may be deemed valid in Time Division Duplex (TDD) systems, when the Uplink (UL) and DL signals are transmitted at the same frequency, provided that the co-channel interference is also similar at the BS and the MSs, although this is not always the case. MUT-aided transmit preprocessing may hence be deemed attractive, when the channel's coherence time is longer than the transmission burst interval. However, for Frequency Division Duplex (FDD) systems, where the UL and DL channels are expected to be different, CIR feedback from the MS's receivers to the BS transmitter is necessary [2].

Minimum Mean-Square-Error (MMSE) MUT is popular owing to its appealing simplicity [2], [3]. However, nonlinear MUT techniques were found to be more powerful in terms of approaching the rate region of dirty paper coding (DPC) [4]. Vector precoding [5], [6] is a promising technique, which achieves excellent BER performance compared to linear preprocessing techniques. A so-called Regularized Perturbation technique was proposed in [6] as an improvment of the vector precoding scheme of [6], which was found to be sub-optimum in the sense of minimizing the MSE. Hence, in [7] the authors derived the MMSE vector precoding solution for transmission in frequency-flat fading multiuser scenarios with the aid of a multi-antenna transmitter. The authors of [8] proposed a vector precoder design where the data vector was perturbed by an arbitrary vector in order to minimize the total MSE. By contrast, in [9] a vector precoding technique was proposed, where a continuous-valued perturbation vector was found in order to minimize the MSE of the received signal. In [10], another MMSE vector precoding technique was proposed, and a $2 \mathrm{~dB}$ gain was obtained in a rapidly fading enviroment, when compared to the vector precoding technique of [6]. Although numerous different MMSE vector precoding techniques were proposed, the solution proposed in [7] was deemed to be the predominant MMSE vector precoding scheme [11]-[13].

However, since the BER is the ultimate system performance indicator, precoding schemes designed by minimizing the MBER criterion are attractive. Linear MBER MUT schemes were proposed in [14], [15], while in [14] a symbol-specific MBER MUT scheme was introduced and a general MBER MUT algorithm was advocated in [15]. An improved nonlinear MBER MUT scheme was proposed in [10] for improving the BER in a quasi-static fading environment, which was based on the assumption that the BER could be expressed as a direct function of the average of MSE, which however does not lead to the true BER expression. Hence, in this paper we derive the BER expression for the system, so that the perturbation vector can be chosen based on the MBER criterion, which facilitates the design of the MBER criterion based vector precoding technique.

The rest of this contribution is structured as follows. In Section II, the model of the downlink SDMA system is introduced. A review of classic vector precoding schemes is provided in Section III, including Zero-Forcing (ZF) vector precoding and MMSE vector precoding. Section IV describes the proposed vector precoding algorithm. Our simulation study is provided in Section V. Finally, we conclude our discourse in Section VI.

\section{System ModeL}

In this section, the system model of Fig. 1 is described, where the DL of a SDMA system is considered. The BS is equipped with $N$ DL transmit antennas and communicates with $K$ MSs, each employing a single receive antenna. The 


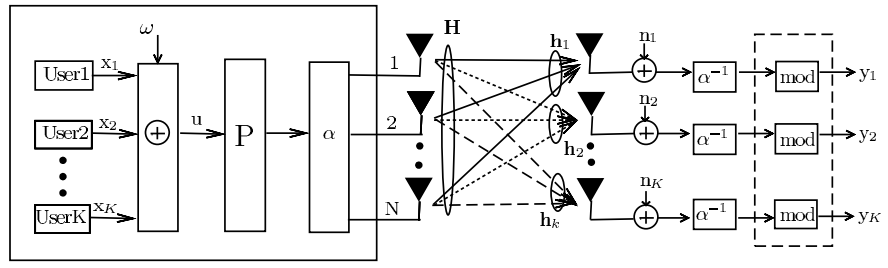

Fig. 1. Schematic diagram of the SDMA system's DL using preprocessing at the BS. The MUT-aided system employs $N$ DL transmit antennas to communicate with $K$ non-cooperative mobile devices.

channel is assumed to be frequency-flat fading and the CSI is assumed to be perfectly known at the BS. The $K$-element vector of information symbols transmitted in the DL to $K$ users is given by $\mathbf{x}=\left[\begin{array}{lll}x_{1} & x_{2} \cdots x_{K}\end{array}\right]^{T}$, where $x_{k}$ denotes the transmitted symbol of the $k$ th MS and the symbol energy is given by $E\left[\left|x_{k}\right|^{2}\right]=\sigma_{x}^{2}$, for $1 \leq k \leq K$, with $E[\bullet]$ denoting the expectation operator. The original transmitted symbol vector $\mathbf{x}$ is then perturbed generating the perturbed vector $\mathbf{u}$ having $K$ elements as given by

$$
\mathbf{u}=\mathbf{x}+\omega
$$

where $\omega$ is a complex-valued perturbation vector, which can be appropriately chosen in order to minimize the total transmission power as in [6], to minimize the MSE as in [7], or to minimize BER as in this paper. More detail about $\omega$ is provided in Section III. The Additive White Gaussian Noise (AWGN) vector $\mathbf{n}$ is defined by $\mathbf{n}=\left[n_{1} n_{2} \cdots n_{K}\right]^{T}$, where $n_{k}, 1 \leq k \leq K$ is a complex-valued Gaussian random process with zero mean and a variance of $\sigma_{n}^{2}$ per real dimension. The channel matrix $\mathbf{H}$ is given by

$$
\mathbf{H}=\left[\begin{array}{llll}
\mathbf{h}_{1} & \mathbf{h}_{2} & \cdots & \mathbf{h}_{K}
\end{array}\right],
$$

where $\mathbf{h}_{k}=\left[\begin{array}{ll}h_{1, k} & h_{2, k} \cdots h_{N, k}\end{array}\right]^{T}, 1 \leq k \leq K$, is the $k$ th user's spatial signature. The channel taps $h_{i, k}$ for $1 \leq k \leq$ $K$ and $1 \leq i \leq N$ are independent of each other and obey the complex-valued Gaussian distribution associated with $E\left[\left|h_{i, k}\right|^{2}\right]=1$. The $(N \times K)$-element precoding matrix $\mathbf{P}$ is given by

$$
\mathbf{P}=\left[\begin{array}{lll}
\mathbf{p}_{1} & \mathbf{p}_{2} & \cdots \\
\mathbf{p}_{K}
\end{array}\right],
$$

where $\mathbf{p}_{k}, 1 \leq k \leq K$ is the precoder's coefficient vector for the $k$ th user's data stream. Given a fixed total transmit power $\mathrm{E}_{T}$ at the BS, an appropriate scaling factor should be used to fullfill this transmit power constraint, which is defined as

$$
\alpha=\sqrt{\frac{\mathrm{E}_{T}}{E\left[\|\mathbf{P u}\|^{2}\right]}} .
$$

At the receiver, the reciprocal of the scaling factor, namely $\alpha^{-1}$, is used to scale the received signal in order to maintain unity-gain transmission.

Let $\hat{y}_{k}, 1 \leq k \leq K$ denote the received signal of the $k$ th user before the modulo operation defined in [6], which will be described in detail in Section III. Then the modulo operation invoked for $\hat{y}_{k}$, in order to remove the effect of the specifically chosen perturbation vector of Eq.(1), can be described as

$$
\bmod _{\tau}\left(\hat{y}_{k}\right)=\hat{y}_{k}-\left\lfloor\frac{\Re\left[\hat{y}_{k}\right]+\tau / 2}{\tau}\right\rfloor \tau-i\left\lfloor\frac{\Im\left[\hat{y}_{k}\right]+\tau / 2}{\tau}\right\rfloor \tau \text {. }
$$

where we have $i^{2}=-1$ and $\lfloor\bullet\rfloor$ denotes the largest integer less than or equal to its argument.

Therefore, the received signal can be described as

$$
\mathbf{y}=\bmod _{\tau}\left(\mathbf{H}^{T} \mathbf{P u}+\alpha^{-1} \mathbf{n}\right),
$$

where $\mathbf{y}=\left[\begin{array}{lll}y_{1} & y_{2} \cdots y_{K}\end{array}\right]^{T}$ denotes the received signal vector after the modulo operation and $y_{k}, 1 \leq k \leq K$ is directly used for the $k$ th MT to detect the transmitted data symbol $x_{k}$.

\section{Vector Precoding Preliminaries}

In this section, both the $\mathrm{ZF}$ vector precoding and the MMSE vector precoding will be re-visited briefly. The $\mathrm{ZF}$ vector precoder first generates the pseudo-inverse of the channel matrix, which means that the precoding matrix $\mathbf{P}$ in Fig. 1 is chosen to be [6]

$$
\mathbf{P}=\mathbf{H}^{H}\left(\mathbf{H} \mathbf{H}^{H}\right)^{-1} .
$$

As the second step, it imposes a discrete perturbation vector on the $K$-user data vector $\mathbf{x}$ in order to minimize the required transmission power, which can then be removed by a simple modulo operation employed at the receiver. To elabarate a little further, the modulo operator of Fig. 1 maps any element of the received signal vector into the fundamental Voronoi region of $\nu=\{a+i b \mid a, b \in[-\tau / 2, \tau / 2)\}$. The value of $\tau$ is a positive real number determined by the modulation constellation employed, which was suggested by the authors of [6] based on their simulations to be chosen according to [6]

$$
\tau=2\left(|c|_{\max }+\Delta / 2\right),
$$

where $|c|_{\text {max }}$ is the absolute value of the modulated symbol having the largest magnitude, and $\Delta$ is the spacing between the constellation points seen in Fig. 2.

Then, a certain degree of freedom is provided at the transmitter to choose any of the phasors represented by the same symbol in Fig. 2, which can represent the same original 4-QAM data [7]. Therefore, the original 4-QAM constellation can be seen to be periodically extended, as examplified in Fig. 2.

More specifically, consider the original 4-QAM scheme of Fig. 2 having the amplitude of $\{ \pm 1 / 2\}^{2}$ for the complexvalued model. The values of $|c|_{\max }$ and $\Delta$ are $|c|_{\max }=\frac{1}{2}$ and $\Delta=1$, respectively. Hence, we have $\tau=2$, according to Eq.(8).

In both the ZF and MMSE vector precoding schemes of [6] and [7], the perturbation vector $\omega$ is chosen to be [6]

$$
\omega=\tau \zeta
$$

where $\zeta$ is a complex vector whose components are $a+i b$, where $a$ and $b$ are integers. 


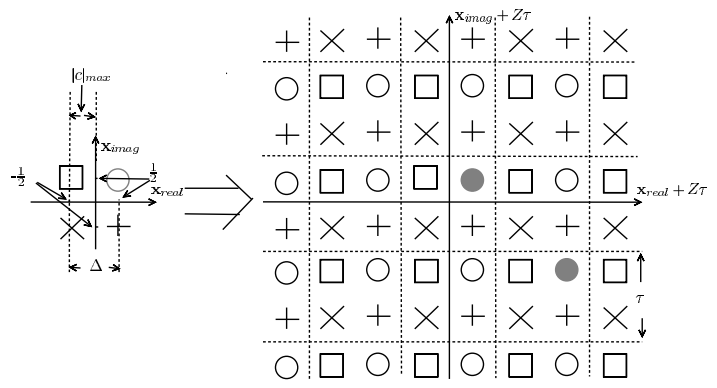

Fig. 2. Standard 4-QAM constellation and the periodically extended 4-QAM constellation when using vector precoding. All symbols from the extended constellation with the same marker represent the same data [16]. The filled points of $0.5+0.5 i$ and $2.5-1.5 i$ are plotted in grey for illustration.

Then the perturbation vector is chosen by minimizing the transmit power as [6]

$$
\zeta_{Z F}=\arg \min _{\zeta}\left\|\mathbf{H}^{H}\left(\mathbf{H} \mathbf{H}^{H}\right)^{-1}(\mathbf{x}+\tau \zeta)\right\|^{2} .
$$

Suppose $\zeta$ is found to be $\zeta=[1-i]$ when the original information symbol is $\mathbf{x}=[0.5+0.5 i]$, according to Eq.(10). Then, the discrete perturbation vector of Eq.(9) becomes $\omega=$ $\tau \zeta=[2-2 i]$. Hence, the perturbed transmitted signal in Fig 2 becomes $\mathbf{u}=\mathbf{x}+\omega=[2.5-1.5 i]$.

Assuming that transmissions take place over an ideal channel, hence the received signal is $\hat{\mathbf{y}}=[2.5-1.5 i]$, then taking the modulo function according to Eq.(5) we have $\mathbf{y}=[0.5+0.5 i]$, which is the same as the transmitted signal $\mathbf{x}$. This example demonstrated that the perturbed constellation points of $2.5-1.5 i$ and $0.5+0.5 i$ seen in Fig. 2 indeed represent the same data, when taking the modulo function of Eq.(5) into account.

By contrast, if the transmission channel is not ideal, the contaminated received signal may become $\hat{\mathbf{y}}=[2.1-0.9 i]$. Then after the modulo operation of Eq.(5), we have $\mathbf{y}=[0.1-$ $0.9 i]$. Hence, compared to the value of $\mathbf{x}=[0.5+0.5 i]$, we can see that a quadrature-phase component error occours. This also shows that the received signal is mapped into the fundamental Voronoi region of $\nu=\{a+i b \mid a, b \in[-\tau / 2, \tau / 2)\}$ after the modulo operation.

By contrast, for MMSE vector precoding, the precoding matrix $\mathbf{P}$ is [7]

$$
\mathbf{P}=\mathbf{H}^{H}\left(\mathbf{H H}^{H}+\beta \mathbf{I}_{c}\right)^{-1},
$$

where $\beta=\operatorname{Tr}\left(\mathbf{R}_{n n}\right)$, while $\mathbf{R}_{n n}$ is the noise covariance matrix, $\operatorname{Tr}(\bullet)$ denotes the trace of the matrix and $\mathbf{I}_{c}$ is the complex-valued identity matrix.

It was shown in [7] that the MMSE perturbation vector should be found by the following search procedure [7]

$$
\zeta_{M M S E}=\arg \min _{\zeta}\|\mathbf{L}(\mathbf{x}+\tau \zeta)\|^{2},
$$

where the upper triangular matrix $\mathbf{L}$ is derived with the aid of the Cholesky decomposition of $\left(\mathbf{H H}^{H}+\beta \mathbf{I}_{c}\right)^{-1}$, given by $\left(\mathbf{H} \mathbf{H}^{H}+\beta \mathbf{I}_{c}\right)^{-1}=\mathbf{L}^{H} \mathbf{L}$.

The searches for the optimal perturbation vector in both $\mathrm{ZF}$ and MMSE vector precoding schemes were respectively suggested in [6] and [7] to be implemented by using the socalled sphere encoding algorithm [6]

\section{MBER CRITERION FOR VECTOR PRECODING FOR 4-QAM}

When the modulo operation of Eq.(5) is employed at the receivers, given the 4-QAM DL symbol vector $\mathbf{x}$, as well as the continuous-valued perturbed vector $\mathbf{u}$ of Eq.(1) and the factor $\tau$ of Eq.(8), the Probability of Error (PE) encountered at the output of the receiver after the modulo operation of the in-phase component of user $k$ can be expressed as

$$
P_{e_{I}, k}=P\left\{\operatorname{sgn}\left(\Re\left[x_{k}\right]\right) \cdot \Re\left[y_{k}\right]<0\right\},
$$

where $y_{k}$ is defined in Eq.(6).

Let us define $s_{2}=\operatorname{sgn}\left(\Re\left[x_{k}\right]\right) \cdot \Re\left[y_{k}\right]$ as a signed decision variable and $c_{R}=\operatorname{sgn}\left(\Re\left[x_{k}\right]\right) \cdot \bmod _{\tau}\left(\Re\left[\left(\mathbf{h}_{k}^{T} \mathbf{P u}\right)\right]\right)$, where $\bmod _{\tau}\left(\Re\left[\left(\mathbf{h}_{k}^{T} \mathbf{P u}\right)\right]\right)$ denotes the noiseless signal at the output of the receiver after the modulo operation associated with user $k$. The PDF of $s_{2}$, which can be seen in Fig. 3, is then given by

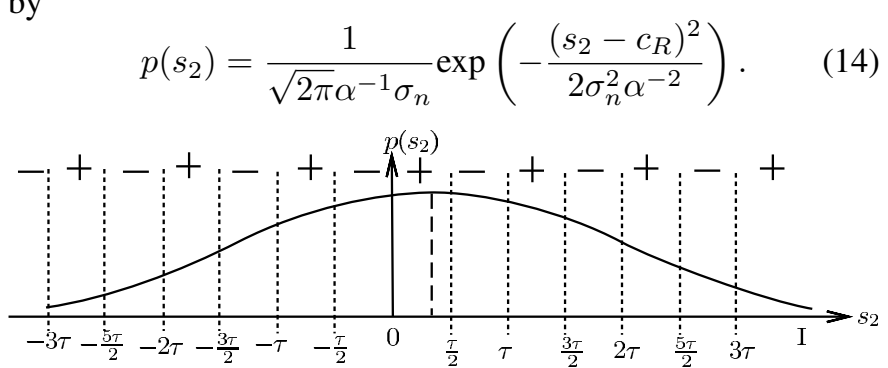

Fig. 3. The PDF of $s_{2}$.

As we stated above, the in-phase component of the received signal vector associated with user $k$ is mapped by the modulo operator into the interval of $[-\tau / 2, \tau / 2)$. For example, if $\operatorname{sgn}\left(\Re\left[x_{k}\right]\right)>0, \Re\left[\hat{y}_{k}\right]=1.75 \tau$, it is then mapped to $\Re\left[y_{k}\right]=-0.25 \tau$ after the modulo operation. Because we have $s_{2}=\operatorname{sgn}\left(\Re\left[x_{k}\right]\right) \cdot \Re\left[y_{k}\right]<0$, a decision error occurs. In other words, the decision areas can be interpreted to be periodically extended for $s_{2}$, as in Fig3. Hence, for example, when $s_{2}$ falls into the interval of $\left[-\frac{5 \tau}{2},-2 \tau\right),\left[-\frac{3 \tau}{2},-\tau\right)$, $\left[-\frac{\tau}{2}, 0\right),\left[\frac{\tau}{2}, \tau\right),\left[\frac{3 \tau}{2}, 2 \tau\right)$ and $\left[\frac{5 \tau}{2}, 3 \tau\right)$, a decision error occurs. Under the assumption that the PE in the region of $(3 \tau,+\infty)$ is identical to the probability of the signal residing in the interval between $(-\infty,-3 \tau)$, which is always true owing to the symmetry of the PDF, the PE can be approximated as [17]

$$
\begin{aligned}
P_{e_{I}, k} & \approx \int_{-\infty}^{-3 \tau} p\left(s_{2}\right) d s_{2}+\int_{-\frac{5 \tau}{2}}^{-2 \tau} p\left(s_{2}\right) d s_{2} \\
& +\int_{-\frac{3 \tau}{2}}^{-\tau} p\left(s_{2}\right) d s_{2}+\int_{-\tau}^{0} p\left(s_{2}\right) d s_{2} \\
& +\int_{\frac{\tau}{2}}^{\tau} p\left(s_{2}\right) d s_{2}+\int_{\frac{3 \tau}{2}}^{2 \tau} p\left(s_{2}\right) d s_{2} \\
& +\int_{\frac{5 \tau}{2}}^{3 \tau} p\left(s_{2}\right) d s_{2} .
\end{aligned}
$$


Then, $P_{e_{I}, k}$ can be further expressed as

$$
\begin{aligned}
P_{e_{I}, k} & =Q\left(\frac{c_{R}+3 \tau}{\alpha^{-1} \sigma_{n}}\right)+Q\left(\frac{-\frac{5 \tau}{2}-c_{R}}{\alpha^{-1} \sigma_{n}}\right)-Q\left(\frac{-2 \tau-c_{R}}{\alpha^{-1} \sigma_{n}}\right) \\
& +Q\left(\frac{-\frac{3 \tau}{2}-c_{R}}{\alpha^{-1} \sigma_{n}}\right)-Q\left(\frac{-\tau-c_{R}}{\alpha^{-1} \sigma_{n}}\right)+Q\left(\frac{-\frac{\tau}{2}-c_{R}}{\alpha^{-1} \sigma_{n}}\right) \\
& -Q\left(\frac{-c_{R}}{\alpha^{-1} \sigma_{n}}\right)+Q\left(\frac{\frac{\tau}{2}-c_{R}}{\alpha^{-1} \sigma_{n}}\right)-Q\left(\frac{\tau-c_{R}}{\alpha^{-1} \sigma_{n}}\right) \\
& +Q\left(\frac{\frac{3 \tau}{2}-c_{R}}{\alpha^{-1} \sigma_{n}}\right)-Q\left(\frac{2 \tau-c_{R}}{\alpha^{-1} \sigma_{n}}\right)+Q\left(\frac{\frac{5 \tau}{2}-c_{R}}{\alpha^{-1} \sigma_{n}}\right) \\
& -Q\left(\frac{3 \tau-c_{R}}{\alpha^{-1} \sigma_{n}}\right) .
\end{aligned}
$$

Hence, the average PE of the in-phase component of $\mathbf{y}$ at the receiver is given by

$$
P_{e_{I}, \mathbf{x}}=\frac{1}{K} \sum_{k=1}^{K}\left(P_{e_{I}, k}\right) .
$$

Similarly, let $c_{I}=\operatorname{sgn}\left(\Im\left[x_{k}\right]\right) \cdot \bmod _{\tau}\left(\Im\left[\left(\mathbf{h}_{k}^{T} \mathbf{P u}\right)\right]\right)$. Then the PE of the quadrature-phase component for the $k$ th user is given by

$$
\begin{aligned}
P_{e_{Q}, k} & =Q\left(\frac{c_{I}+3 \tau}{\alpha^{-1} \sigma_{n}}\right)+Q\left(\frac{-\frac{5 \tau}{2}-c_{I}}{\alpha^{-1} \sigma_{n}}\right)-Q\left(\frac{-2 \tau-c_{I}}{\alpha^{-1} \sigma_{n}}\right) \\
& +Q\left(\frac{-\frac{3 \tau}{2}-c_{I}}{\alpha^{-1} \sigma_{n}}\right)-Q\left(\frac{-\tau-c_{I}}{\alpha^{-1} \sigma_{n}}\right)+Q\left(\frac{-\frac{\tau}{2}-c_{I}}{\alpha^{-1} \sigma_{n}}\right) \\
& -Q\left(\frac{-c_{I}}{\alpha^{-1} \sigma_{n}}\right)+Q\left(\frac{\frac{\tau}{2}-c_{I}}{\alpha^{-1} \sigma_{n}}\right)-Q\left(\frac{\tau-c_{I}}{\alpha^{-1} \sigma_{n}}\right) \\
& +Q\left(\frac{\frac{3 \tau}{2}-c_{I}}{\alpha^{-1} \sigma_{n}}\right)-Q\left(\frac{2 \tau-c_{I}}{\alpha^{-1} \sigma_{n}}\right)+Q\left(\frac{\frac{5 \tau}{2}-c_{I}}{\alpha^{-1} \sigma_{n}}\right) \\
& -Q\left(\frac{3 \tau-c_{I}}{\alpha^{-1} \sigma_{n}}\right) .
\end{aligned}
$$

Then, the average PE for the quadrature-phase component of $\mathbf{y}$ at the receiver of the $K \mathrm{MSs}$ is given by

$$
P_{e_{Q}, \mathbf{x}}=\frac{1}{K} \sum_{k=1}^{K}\left(P_{e_{Q}, k}\right) .
$$

Thus, the resultant PE for 4-QAM signalling becomes

$$
P_{e, \mathbf{x}}=\left(P_{e_{I}, \mathbf{x}}+P_{e_{Q}, \mathbf{x}}\right) / 2 .
$$

Hence, given the precoding matrix $\mathbf{P}$ of Fig. 1, the optimum continuous-valued perturbation vector $\omega$ is found by satisfying the following equation

$$
\omega_{o p t}=\arg \min _{\omega} P_{e, \mathbf{x}} .
$$

It should also be pointed out that the continuous-valued perturbation in Eq.(1) can be viewed as a discrete perturbation vector modified by another continuous-valued perturbation vector according to

$$
\omega=\tau \zeta+\phi
$$

where $\phi$ is a continuous-valued perturbation vector satisfying $\|\Re(\phi)\|<\frac{\tau}{2}$ and $\|\Im(\phi)\|<\frac{\tau}{2}$.
Therefore, in our proposed vector precoding scheme, if only discrete perturbations are allowed, the complexity imposed is identical to that of the MMSE vector precoding. Accordingly, it will be demonstrated that the performance of the vector precoding we proposed using discrete perturbation is similar to that of the MMSE vector precoder.

\section{Simulation Results}

In our simulations, the precoding matrix was chosen to be [7]

$$
\mathbf{P}=\mathbf{H}^{H}\left(\mathbf{H} \mathbf{H}^{H}+\beta \mathbf{I}_{c}\right)^{-1} \text {. }
$$

We used the square-constellation based 4-QAM scheme having the amplitude of $\{ \pm 1 / 2\}^{2}$ for the complex-valued model. The value of $\tau$ for the ZF vector precoding, the MMSE vector precoding and our proposed vector precoding schemes was chosen to be $\tau=2$.

The DL of a multiuser system employing $N=4$ transmit antennas at the BS to support $K=4$ 4-QAM users was considered. Perfect CSI knowledge was assumed at the BS for transmission over the $(K \times N)$-element flat Rayleigh fading MIMO channels. The received signals after the modulo operation are directly used for making decisions.

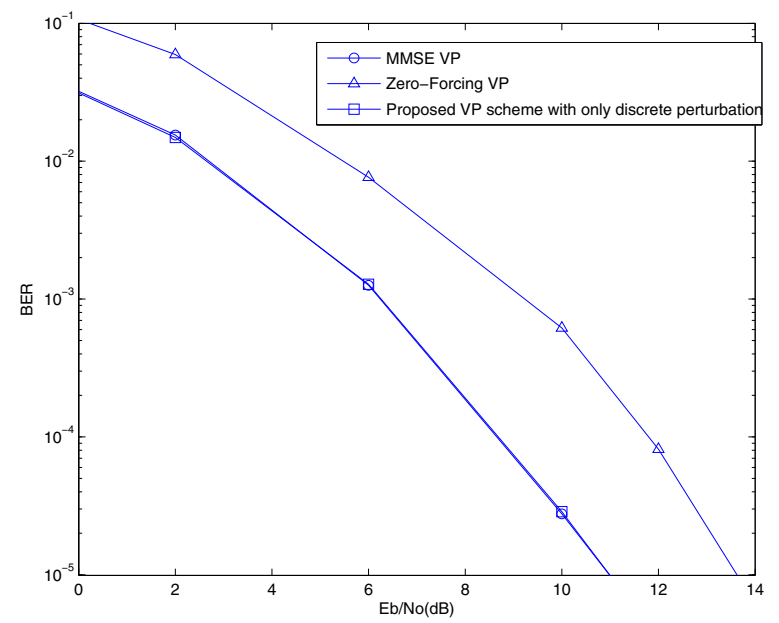

Fig. 4. Uncoded BER versus SNR performance of the ZF vector precoding algorithm proposed in [6], of the MMSE vector precoder of [7] and of our proposed vector precoding algorithm, when only discrete perturbation is allowed, while using the modulo operation at the receivers for communicating over flat Rayleigh fading channels using $N=4$ transmit antennas to support $K=4$ 4-QAM users.

When the modulo operation Eq.(5) is employed at the receivers, the uncoded $\mathrm{BER}$ performance of the $\mathrm{ZF}$ vector precoding algorithm proposed in [6], of the MMSE vector precoding of [7] and of our proposed vector precoding algorithm using only discrete perturbation are compared in Fig. 4. As we can see from the figure, the proposed vector precoding design outperforms the $\mathrm{ZF}$ vector precoding scheme and its performance is identical to that of the MMSE vector precoder. Therefore, we may conclude that the proposed vector 


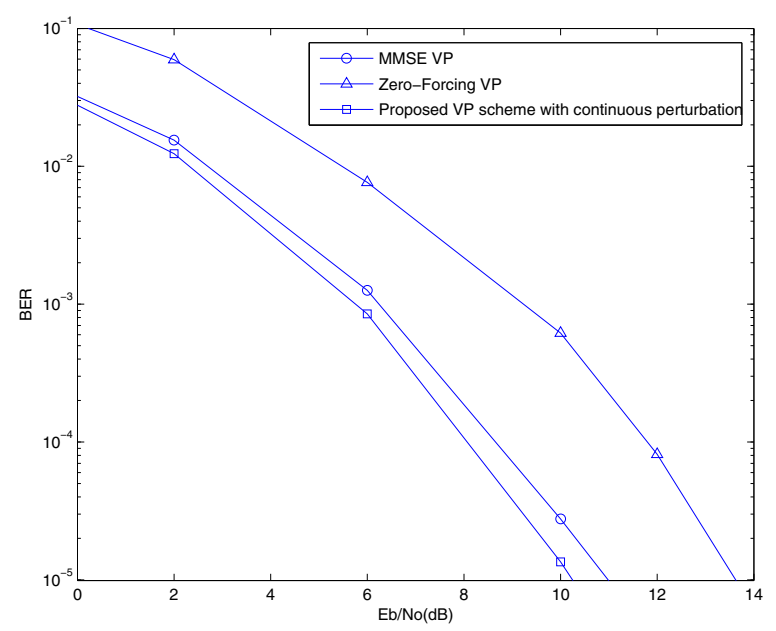

Fig. 5. Uncoded BER versus SNR performance of the ZF vector precoding algorithm proposed in [6], of the MMSE vector precoder in [7] and of our proposed vector precoding algorithm, when continuous perturbation is allowed, while using the modulo operation at the receivers for communicating over flat Rayleigh fading channels using $N=4$ transmit antennas to support $K=4$ 4-QAM users.

precoding scheme using only discrete perturbation achieves the same BER performance as that of the MMSE vector precoding algorithm.

Furthermore, no continuous-valued perturbation vector can be found for MMSE vector precoding in order to improve its performance. However, in our proposed vector precoder design, a continuous-valued perturbation vector can be used in order to achieve an improved performance by exploring the associated extra degree of freedom in terms of choosing a continuous-valued perturbation vector, as demonstrated in Fig. 5. As we can see from the figure, our proposed vector precoding design outperforms both the ZF and MMSE vector precoding schemes at all SNRs. More explicitly, when compared to ZF vector precoding, our proposed algorithm achieved a $3.5 \mathrm{~dB}$ SNR gain at the target BER of $10^{-5}$, and an SNR gain of about $0.8 \mathrm{~dB}$ over MMSE vector precoding at the same target BER.

However, we found in our simulations that the search for the optimum continuous perturbation vector results in a challenging nonconvex optimization problem, where numerous local minima exist. In our approach, we use the results of MMSE vector precoding as the starting points during the optimization process, hence the complexity of our proposed vector precoder design using a continuous-valued perturbation vector scheme is higher than that of the MMSE vector precoding scheme. The complexity reduction of the proposed vector precoding scheme requires further research.

\section{CONCLUSIONS}

In this paper, we proposed a novel vector precoding scheme based on the MBER criterion as an improvement to the MMSE vector precoding scheme. It was shown that the proposed vector precoding scheme achieves the same BER performance as the MMSE vector precoder at the same complexity, when only discrete vector perturbations are allowed. However, the proposed precoder's performance can be further improved when continuous-valued vector perturbations are carried out.

Our future work is related to the analysis of our proposed vector precoding design in the presence of channel estimation errors as well as in diverse realistic propagation scenarios, when protected by soft-decision-aided channel codes.

\section{REFERENCES}

[1] L.-L. Yang, "Design of linear multiuser transmitters from linear multiuser receivers," in Proceedings of ICC 2007, (Glasgow, UK), pp. 5258 - 5263, June 24-28, 2007.

[2] D. Yang, L.-L. Yang and L. Hanzo, "Performance of SDMA systems using transmitter preprocessing based on noisy feedback of vectorquantized channel impulse responses," in Proceedings of VTC2007Spring, (Dublin, Ireland), pp. 2119-2123, Apr. 22-25, 2007.

[3] B. R. Vojčić and W. M. Jang, "Transmitter precoding in synchronous multiuser communications," IEEE Transactions on Communications, vol. 46, pp. 1346-1355, Oct. 1998.

[4] M. Costa, "Writing on dirty paper," IEEE Transactions on Information Theory, vol. 29, p. 439 441, May 1983.

[5] C. B. Peel, B. M. Hochwald and A. L. Swindlehurst, "A vectorperturbation technique for near-capacity multiantenna multiuser communication - part I: channel inversion and regularization," IEEE Transactions on Communications, vol. 53, pp. 195-202, Jan. 2005.

[6] B. M. Hochwald, C. B. Peel and A. L. Swindlehurst, "A vectorperturbation technique for near-capacity multiantenna multiuser communication - part II: perturbation," IEEE Transactions on Communications, vol. 53, pp. 537-544, Mar. 2005.

[7] D. A. Schmidt, M. Joham and W. Utschick, "Minimum mean square error vector precoding," in Proceedings of PIMRC 2005, vol. 1, pp. 107 -111 , Sep. 2005.

[8] E. Y. Kim and J. Chun, "Optimum vector perturbation minimizing total MSE in multiuser MIMO downlink," in Proceedings of ICC 2007, pp. 4242 - 4247, June 2006.

[9] W. S. Chua, C. Yuen and F. Chin, "A continuous vector-perturbation for multi-antenna multi-user communication," in Proceedings of VTC2007Spring, (Dublin, Ireland), pp. 1806 - 1810, Apr. 22-25, 2007.

[10] A. Callard, A. Khandani, and A. Saleh, "Vector precoding with MMSE for the fast fading and quasi-static multi-user broadcast channel," in Proceedings of CISS 2006, pp. 1002 - 1007, Mar. 2006.

[11] F. Liu, L. Jiang and C. He, "Low complexity MMSE vector precoding using lattice reduction for MIMO systems," in Proceedings of ICC 2007, pp. 2598 - 2603, June 2007.

[12] S. S. Christensen and E. de Carvalho, "Achievable sum-rates in MIMO broadcast channels with vector precoding techniques using coded modulation," in Proceedings of VTC2007-Spring, (Dublin, Ireland), pp. 2261 - 2265, Apr. 22-25, 2007.

[13] R. Habendorf and G. Fettweis, "Vector precoding with bounded complexity," in Proceedings of SPAWC 2007, pp. 1 - 5, June 17-20, 2007.

[14] R. Irmer, W. Rave and G. Fettweis, "Minimum BER transmission for TDD-CDMA in frequency-selective channels," in Proceedings of PIMRC 2003, vol. 2, (Beijing, China), pp. 1260-1264, September 7-10, 2003.

[15] A. Hjørungnes and P. S. R. Diniz, "Minimum BER prefilter transform for communications systems with binary signaling and known FIR MIMO channel," IEEE Signal Processing Letters, vol. 12, pp. 234-237, March 2005.

[16] C. Windpassinger, Detection and precoding for multiple input multiple output channels. PhD thesis, University of Erlangen-Nuremberg, Erlangen, Germany, June 2004.

[17] L. Hanzo, S. X. Ng, T. Keller and W. T Webb, Quadrature amplitude modulation: from basics to adaptive trellis-coded, turbo-equalised and space-time coded OFDM, CDMA and MC-CDMA systems. New York, USA: John Wiley, IEEE Press, 2004. 